\title{
Effects of Demographic Stochasticity on Population Persistence in Advective Media
}

\author{
Allison Kolpas ${ }^{\mathrm{a}, \mathrm{b}, *}$, Roger M. Nisbet ${ }^{\mathrm{a}}$ \\ ${ }^{a}$ Department of Ecology, Evolution, and Marine Biology, University of California, \\ Santa Barbara, CA, USA \\ ${ }^{b}$ Present address: Department of Mathematical Sciences, University of Delaware, Newark, \\ DE, USA
}

Received: 29 June 2009 / Accepted: 17 November 2009 / Published online: 5 February 2010 (C) The Author(s) 2010. This article is published with open access at Springerlink.com

\begin{abstract}
Many populations live and disperse in advective media. A fundamental question, known as the "drift paradox" in stream ecology, is how a closed population can survive when it is constantly being transported downstream by the flow. Recent populationlevel models have focused on the role of diffusive movement in balancing the effects of advection, predicting critical conditions for persistence. Here, we formulate an individualbased stochastic analog of the model described in (Lutscher et al., SIAM Rev. 47(4):749$772,2005)$ to quantify the effects of demographic stochasticity on persistence. Population dynamics are modeled as a logistic growth process and dispersal as a position-jump process on a finite domain divided into patches. When there is no correlation in the interpatch movement of residents, stochasticity simply smooths the persistence-extinction boundary. However, when individuals disperse in "packets" from one patch to another and the flow field is memoryless on the timescale of packet transport, the probability of persistence is greatly enhanced. The latter transport mechanism may be characteristic of larval dispersal in the coastal ocean or wind-dispersed seed pods.
\end{abstract}

Keywords Population dynamics · Drift paradox · Demographic stochasticity · Stochastic simulation algorithm

\section{Introduction}

Many populations, including bacteria in the gut, plants with wind or waterborne seeds, and animals in streams, estuaries, and the ocean live and disperse in advective media. A fundamental question in population dynamics is how do such populations persist without any net influx of new settlers when they are constantly being transported downstream or downwind. Such a question is not only interesting from a theoretical standpoint, but

\footnotetext{
*Corresponding author.

E-mail address: akolpas@ math.udel.edu (Allison Kolpas).
} 
has practical application in the design and management of environmental reserves and in other restoration projects (Anderson et al., 2006).

In lotic ecosystems such as streams and rivers, the question of the persistence of a population subject to downstream dispersal has been referred to as the "drift paradox" and is mostly associated with drifting invertebrates such as mayfly, stonefly, caddisfly, and crustaceans which serve as a fish food source and are thus vital to the functioning of the ecosystem (Hershey et al., 1993; Müller, 1974). The same question of persistence has been considered for populations in the coastal ocean subject to tides and long-shore currents (Alexander and Roughgarden, 1996; Byers and Pringle, 2006; Gaines and Bertness, 1992). Many of the proposed resolutions to the drift paradox involve some form of compensatory movement in opposition to the flow as a result of turbulence (Speirs and Gurney, 2001), biased flight upstream before oviposition (Müller, 1982), or individuals crawling sufficient distances against the flow along the benthos (Humphries and Ruxton, 2002). Mathematical models has become a useful tool for evaluating the utility of such hypotheses and suggesting new ones.

Recent models in the form of integrodifferential equations and reaction-advectiondiffusion equations have focused on the role of diffusive movement, whether it be due to turbulence in the flow or individuals crawling upstream, in balancing the effects of advection for populations with a single life stage (Ballyk and Smith, 1999; Lutscher et al., 2005; Pachepsky et al., 2005; Speirs and Gurney, 2001). Such models have shown that if the rate of settlement upstream is sufficiently large to balance the loss due to death and downstream movement, then a population can persist on a sufficiently large domain. Predictions regarding the critical flow speed or domain size can be made given a suitable model for the growth and reproduction of the population. Indeed, the question of a "critical domain size" dates back to Skellam's work in the 1950s on reaction-diffusion equations for the logistic growth of populations on bounded domains (Skellam, 1951). Such critical domain size calculations involve linearizing the governing equations about the zero steady state and solving a Sturm-Liouville problem to find conditions under which it is unstable (Okubo, 1980).

Although there has been much recent interest in population dynamics in advective media, most models are deterministic and, therefore, do not address what effect, if any, stochasticity has on persistence. For populations dispersing in advective media, stochasticity can be attributed to variability in reproduction rates, movement rates, and distances traveled by individuals. In addition, correlations in the dispersal activity of individuals can greatly impact their density and thus the odds of persistence. An example of the latter is large-scale eddy events in the coastal ocean which intermittently sweep larvae in groups from one place to another. The location and timing of such events is highly variable within a given spawning season and from season to season (Siegel et al., 2008). Another example is wind-dispersed seed pods in a heterogeneous landscape.

It is well known that demographic stochasticity can have profound consequences in small populations (Gurney and Nisbet, 1998). For example, consider a population containing an integer number of individuals undergoing a simple birth-death process. If the death rate exceeds the birth rate, extinction is unavoidable both deterministically and in the stochastic model. However, when the birth rate exceeds the death rate, the deterministic theory predicts persistence, while in the stochastic model the probability of extinction may be nonnegligible for populations initially small in number. Thus, a critical condition 
for persistence for a deterministic model may not carry over when demographic stochasticity is introduced.

Here, we introduce a discrete-state continuous-time stochastic analog of the integrodifferential equation developed in Lutscher et al. (2005) for the dynamics of a population dispersing in advective media. The model allows us to explore the effects of demographic stochasticity, both in reproduction and movement, as well as correlations in the dispersal activity of individuals, on population persistence. The paper is organized as follows. In Section 2, we briefly review the integrodifferential model, rewrite the system in terms of nondimensional quantities, and discuss conditions on the flow speed and domain size for persistence for different forms of boundary conditions under our parametrization. In Sections 3 and 4, we formulate the corresponding stochastic model, present the algorithms used to simulate the stochastic population dynamics, and analyze the results. Finally, in Section 5, we discuss the implications of our results for the persistence of populations in advective media.

\section{The model}

\subsection{Population dynamics}

We consider the integrodifferential equation introduced in Lutscher et al. (2005) for the spatio-temporal dynamics of a population in a one-dimensional domain. We focus our attention on benthic organisms in a lotic environment such as a stream or river reach. The model can be derived from the two-compartment drift-benthos (D-B) model formulated in Pachepsky et al. (2005) as follows. Let $p(x, t)$ and $w(x, t)$ be the population density at location $x \in[0, L]$ along the benthos and drift, respectively, at time $t$. Furthermore, suppose the benthic population grows logistically, the drifting population disperses via an advection-diffusion process, and transfer between the two compartments occurs via a Poisson process. Then the evolution of $p$ and $w$ is governed by the following system of partial differential equations (Pachepsky et al., 2005):

$$
\begin{aligned}
& \frac{\partial w}{\partial t}=\mu p-\alpha w-v \frac{\partial w}{\partial x}+D \frac{\partial^{2} w}{\partial x^{2}}, \\
& \frac{\partial p}{\partial t}=r p\left(1-\frac{p}{K}\right)-\mu p+\alpha w
\end{aligned}
$$

where $r$ is the intrinsic growth rate, $K$ is the local carrying capacity, $\mu$ is the dispersal rate, $\alpha$ is the settlement rate, $v$ is the velocity of the medium (e.g., the wind speed or flow speed), and the diffusion constant $D$ represents the variability in speed.

Now, suppose the settlement rate, $\alpha$, is much larger than the dispersal rate, $\mu$. This means that the characteristic time scale associated with drifting, $1 / \alpha$, is much less than the characteristic time scale associated with residing on the benthos, $1 / \mu$. In the limit that $\epsilon=\mu / \alpha \rightarrow 0 ; v \rightarrow \infty ; D \rightarrow \infty$ with $\epsilon v$ and $\epsilon D$ finite, one may reduce the system to a single integrodifferential equation for the benthic population (Lutscher et al., 2005):

$$
\frac{\partial p}{\partial t}(x, t)=r p(x, t)\left(1-\frac{p(x, t)}{K}\right)-\mu p(x, t)+\mu \int_{0}^{L} k(x, y) p(y, t) d y .
$$


In the model, a population grows logistically on the benthos and movement takes place as a position-jump process with dispersal kernel, $k(x, y)$, giving the probability distribution of stopping points along the benthos from an initial location $y$.

\subsection{Dispersal kernel}

To derive the dispersal kernel, consider the fast-scale movement dynamics in the drift as decoupled from the slow-scale population dynamics on the benthos. Specifically, let $w(t, x ; y)$ be the distribution of individuals in the drift who enter at time $t=0$ from an initial starting point $y$. Then the movement dynamics are described by an advectiondiffusion process with constant settlement rate $\alpha$

$$
\frac{\partial w}{\partial t}=-\alpha w-v \frac{\partial w}{\partial x}+D \frac{\partial^{2} w}{\partial x^{2}},
$$

and with initial density $w(0, x ; y)=\delta(x-y)$. The dispersal kernel is related to the distribution of drifters by $k(x, y)=\int_{0}^{\infty} \alpha w(t, x ; y) d t$. On an infinite domain, the kernel takes the form of a two-sided exponential distribution (Lutscher et al., 2005)

$$
k(x-y) \equiv k(\xi)= \begin{cases}A e^{a_{1} \xi}, & \xi \leq 0 \\ A e^{a_{2} \xi}, & \xi \geq 0\end{cases}
$$

where

$$
A=\frac{\alpha}{\sqrt{v^{2}+4 \alpha D}}, \quad a_{1,2}=\frac{v}{2 D} \pm \sqrt{\frac{v^{2}}{4 D^{2}}+\frac{\alpha}{D}} .
$$

On a finite domain, the dispersal kernel depends on the precise form of the boundary conditions for (3).

As in Lutscher et al. (2005), the kernel is not normalized. Thus, the dispersal process results in additional mortality. First, consider movement not altered at the domain boundary. An example of this would be a no-fishing zone or plants with wind or waterborne seeds. Under these conditions, the dispersal kernel is equal to the kernel derived for an infinite domain but cut off at the domain boundaries (Lutscher et al., 2005).

As a second example, consider the case of a domain with a source at the top $(x=0)$ and a sink at the bottom $(x=L)$. In this case, one may impose a no-flux boundary condition at the top and an absorbing boundary at the bottom:

$$
v w(t, 0)-D\left(\frac{\partial w}{\partial x}\right)_{x=0}=0, \quad w(t, L)=0 .
$$

The solution to this boundary value problem can be found in the Appendix of Lutscher et al. (2005). It is important to note in this case that the kernel is anisotropic, i.e., $k(x, y) \neq$ $k(x-y)$.

\subsection{Nondimensionalization}

We nondimensionalize the model as follows. Let $t^{\prime}=t / t_{s}, x^{\prime}=x / x_{s}$, and $w^{\prime}=x_{s} w$ with $t_{s}=1 / r$ and $x_{s}=\sqrt{\frac{D}{r}}$. Then the dynamics in the drift can be expressed in terms of the 
nondimensional variables $t^{\prime}, x^{\prime}$, and $w^{\prime}$ as

$$
\frac{\partial w^{\prime}}{\partial t^{\prime}}=-\tilde{\alpha} w^{\prime}-\tilde{v} \frac{\partial w^{\prime}}{\partial x^{\prime}}+\frac{\partial^{2} w^{\prime}}{\partial x^{\prime 2}}
$$

where $\tilde{v}=\frac{v}{\sqrt{D r}}$ and $\tilde{\alpha}=\alpha / r$. With this change of variables, and dropping the prime notation, the nondimensional dispersal kernel is given by $\tilde{k}(x, y)=\int_{0}^{\infty} \tilde{\alpha} w(t, x ; y) d t$ and on an infinite domain can be written as

$$
\tilde{k}(\xi)= \begin{cases}\tilde{A} e^{\tilde{a_{1} \xi}}, & \xi \leq 0 \\ \tilde{A} e^{\tilde{a_{2} \xi}}, & \xi \geq 0\end{cases}
$$

where

$$
\tilde{a}_{1,2}=x_{s} a_{1,2}=\frac{\tilde{v}}{2} \pm \sqrt{\frac{\tilde{v}^{2}}{4}+\tilde{\alpha}}, \quad \tilde{A}=x_{s} A=\frac{\tilde{\alpha}}{\sqrt{\tilde{v}^{2}+4 \tilde{\alpha}}} .
$$

This temporal and spatial scaling yields the following rescaled integrodifferential equation for the population dynamics on the benthos:

$$
\frac{\partial p}{\partial t}(x, t)=p(x, t)\left(1-\frac{p(x, t)}{K}\right)-\tilde{\mu} p(x, t)+\tilde{\mu} \int_{0}^{\tilde{L}} \tilde{k}(x, y) p(y, t) d y,
$$

with $\tilde{\mu}=\mu / r$ and $\tilde{L}=L / x_{s}$. Note that $K$ is not scaled out (as it is in Lutscher et al., 2005) because we are interested in the combined effects of demographic stochasticity and nonlinearity on persistence. Population and movement dynamics are controlled by the carrying capacity $K$ and three additional dimensionless quantities: $\tilde{\mu}, \tilde{L}$, and $\tilde{v}$. The first dimensionless quantity, $\tilde{\mu}$, corresponds to the average number of sojourns to the drift made by an individual in time $t_{s}$. Note that $t_{s}$ is the time for a small population to grow by a factor of $e$ and is the same order of magnitude as the doubling time. The dimensionless quantity $\tilde{L}$ is the ratio of the domain length $L$ to $x_{s}=\sqrt{D / r}=\sqrt{D t_{s}}$. Thus, $\tilde{L}$ may be interpreted as a measure of the length of habitat relative to the diffusive spread of the population in time $t_{s}$. The last quantity, $\tilde{v}=\frac{v}{\sqrt{D r}}=\frac{v t_{s}}{\sqrt{D t_{s}}}$, is the reciprocal of the coefficient of variation of the dispersal process in time $t_{s}$. When $\tilde{v}>1$, dispersion is dominated by advection, while when $\tilde{v}<1$ it is dominated by diffusion. Note that in deriving the integrodifferential equation it is assumed there is a separation in time scales in the population and movement dynamics. In this limit, for any dispersal to take place, it is necessary that both $D \rightarrow \infty$ and $v \rightarrow \infty$ as $\mu / \alpha \rightarrow 0$. This ensures that the nondimensional flow velocity $\tilde{v}$ is finite.

\subsection{Stability and critical domain size}

In Lutscher et al. (2005), Pachepsky et al. (2005), the authors derived expressions for the critical domain size and flow speed in terms of parameters related to dispersal and settlement. The critical domain size is defined as the length of habitat below which a population cannot persist while the critical velocity is the flow speed above which no population can persist. An expression relating the two can be derived by linearizing the model equations and determining the stability of the zero steady-state solution from the 
eigenvalues. For the D-B model with $\mu>1$ and a reflecting boundary at the top of the domain and an absorbing boundary at the bottom (Pachepsky et al., 2005), the critical domain size $\tilde{L}_{c}$ and velocity $\tilde{v}_{c}$ are related by the smallest real solution to Pachepsky et al. (2005):

$$
\frac{\sqrt{\frac{4 \tilde{\alpha}}{\tilde{\mu}-1}-\tilde{v}_{c}^{2}}}{\tilde{v}_{c}}+\tan \left(\frac{L_{c}}{2} \sqrt{\frac{4 \tilde{\alpha}}{\tilde{\mu}-1}-\tilde{v}_{c}^{2}}\right)=0 .
$$

When $\alpha \gg \mu$, this expression is a good approximation to the critical domain size for the integrodifferential equation with an anisotropic kernel $k(x, y)$ given by the solution to (3) with boundary conditions (6). The scaled asymptotic velocity, $v^{*}$, above which no population can survive on any length of domain, may be obtained by setting the expression inside the square root of (11) equal to zero and is given by

$$
v^{* 2}=\frac{4 \tilde{\alpha}}{\tilde{\mu}-1} .
$$

In Lutscher et al. (2005), the authors obtained the following expression for $L$ in terms of the dominant eigenvalue $v$ of the integral operator in (2) and the dispersal related constants from the dimensional kernel (4):

$$
L=\frac{4 \tan ^{-1}\left[\left(\sqrt{\frac{4 a_{1}\left|a_{2}\right|}{v\left(a_{1}-a_{2}\right)^{2}}-1}\right)^{-1}\right]}{\left(a_{1}-a_{2}\right) \sqrt{\frac{4 a_{1}\left|a_{2}\right|}{v\left(a_{1}-a_{2}\right)^{2}}-1}} .
$$

The dominant eigenvalue $v$ is related to the dominant eigenvalue $\tilde{v}$ of the integral operator in (10) by $\tilde{v}=\tilde{\mu} v$. Note that expression (13) remains unchanged under our spatial rescaling and is thus satisfied by $\tilde{L}$ and $\tilde{a}_{1,2}$, the latter of which are functions of $\tilde{v}$. The critical domain size $\tilde{L}_{c}$ may be obtained implicitly as a function of $\tilde{v}_{c}$ by setting $\tilde{\mu} v=\tilde{\mu}-1 \Rightarrow v=\frac{\tilde{\mu}-1}{\tilde{\mu}}$ in expression (13). The asymptotic velocity $v^{*}$ can be obtained by setting the denominator of (13) equal to zero and with a little algebra can be shown to be equivalent to expression (12). This is not surprising, since when the length of domain becomes infinite, the precise form of the boundary conditions is not important.

We now consider some specific parameters. Suppose, for example, that $\tilde{\mu}=50$. This corresponds to the case where individuals make on average 50 trips into the drift in the time a small population grows by a factor of $e$ and might be typical of certain invertebrate populations in streams (Elliott, 2002). In addition, suppose $\tilde{\alpha}=1000$ so that $\tilde{\mu} / \tilde{\alpha}=\mu / \alpha=0.05$. This means that individuals spend on average $5 \%$ of their time in the drift and is consistent with the approximation made in the derivation of (2) from the D-B model. For these parameters, $v^{*} \approx 9.04$. In Fig. 1 , we plot the persistence and extinction boundary using expressions (11) and (13). One can see from the figure that in both cases $\tilde{L}$ approaches infinity as $\tilde{v} \rightarrow v^{*}$. When $\tilde{v}=0$ (diffusion dominated case), the minimum critical domain size for population persistence is $\tilde{L}_{c}=0.63$ with movement not altered and $\tilde{L}_{c}=0.35$ for the D-B model with reflecting and absorbing boundaries. Thus, when individuals do not alter movement at the domain boundaries, there is a higher domain size threshold for population persistence. In both cases, decreasing $\tilde{\mu}$ increases $v^{*}$. 


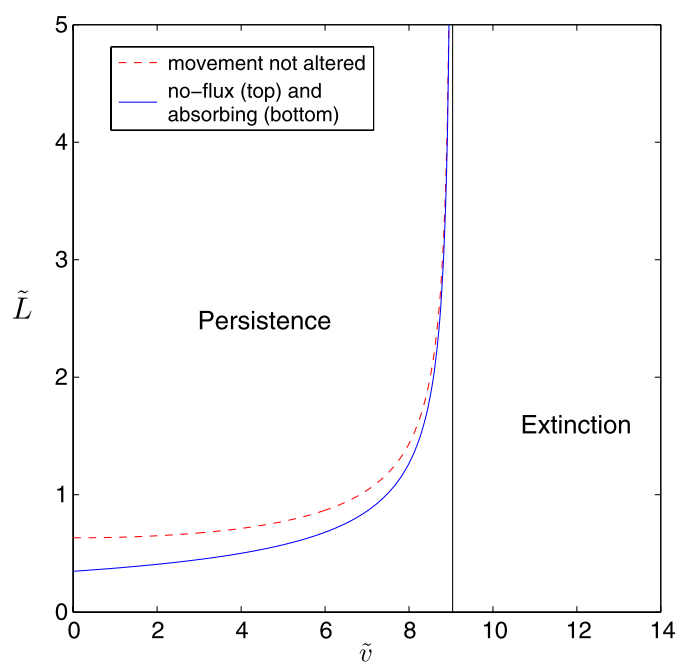

Fig. 1 Critical domain size $\tilde{L}$ as a function of the nondimensional parameter $\tilde{v}$.

\section{Stochastic formulation}

In this section, we formulate a continuous time Markov chain representation of (10) and show how realizations of the stochastic process can be performed using the stochastic simulation algorithm (SSA) (Gillespie, 2007). Stochastic simulations are often the only practical technique available for the study of nonlinear multivariate Markov processes (Gillespie, 2007). We use this stochastic formulation to study the effects of demographic stochasticity on population persistence.

\subsection{Stochastic simulation algorithm}

The SSA was originally developed as an exact procedure for the numerical simulation of the evolution of well-stirred chemically reacting systems in continuous time, but may easily be adapted for the simulation of discrete-population stochastic models arising in ecology. In the stochastic formulation of (10), space is discretized into $N$ patches of width $\Delta x$. The patch width $\Delta x$ sets the size of the domain on which individuals compete for resources. Using the terminology of SSA, we define the state vector, $\mathbf{Z}(t)=\left(Z_{1}(t), Z_{2}(t), \ldots, Z_{N}(t)\right)$, where $Z_{i}(t) \in\{0,1,2, \ldots\}$ is the number of individuals in patch $i$ at time $t \in[0, \infty)$. A change in a local population is a consequence of one of three events: a birth, death, or relocation. Since these three events can occur in any of $N$ different patches, there is a total of $M=3 N$ possible events. The events are mathematically characterized by their propensity functions, $a_{j}(\mathbf{z})$, and state-change vectors, $\boldsymbol{v}_{j}$, for $j=1,2, \ldots, M$. Given $\mathbf{Z}(t)=\mathbf{z}$, the probability that a single event $j$ occurs in a sufficiently small time interval $\Delta t$ is $a_{j}(\mathbf{z}) \Delta t$. Thus, the propensity function gives the stochastic event rate. The state-change vector is the population change vector associated with a single occurrence of an event. If the population is at state $\mathbf{z}$ and event $j$ occurs, $\mathbf{z} \rightarrow \mathbf{z}+\boldsymbol{v}_{j}$. The following table summarizes the propensity functions and state-change vectors associated with a birth, death, and relocation event in patch $i$. 


\begin{tabular}{lll}
\hline Event $($ patch $i)$ & Propensity function & State-change vector \\
\hline Birth & $\tilde{b}_{1} z_{i}(t)+\tilde{b}_{2} z_{i}(t)^{2}$ & $(0, \ldots, 0, \underbrace{+1,}_{i \text { th column }} 0, \ldots, 0)$
\end{tabular}

Death

$$
\tilde{d}_{1} z_{i}(t)+\tilde{d}_{2} z_{i}(t)^{2}
$$

$(0, \ldots, 0, \underbrace{-1,}_{i \text { th column }} 0, \ldots, 0)$

Relocation $\quad \tilde{\mu} z_{i}(t)$

$(0, \ldots, 0, \underbrace{+1,}_{r \text { th column }} 0, \ldots, 0, \underbrace{-1,}_{i \text { th column }} 0, \ldots, 0)$

For this to correspond to the logistic growth process from (10), $\tilde{b}_{1}-\tilde{d}_{1}=1$ and $K^{-1}=$ $\tilde{d}_{2}-\tilde{b}_{2}$. We consider the case that $\tilde{d}_{2}=0$, so that the per capita death rate remains constant while the per capita birth rate is a decreasing function of population size. This implies that $\tilde{b}_{2}=-1 / K$. To ensure the birth rate never becomes negative, we supplement its propensity function with the condition that the birth rate is 0 whenever $z_{i}(t)>b_{1} K$.

In order to simulate sample realizations of the stochastic process, one must generate properly distributed random variables for the time $\tau$ and index $j$ of the next event. It follows from the basic laws of probability that the interevent times are exponentially distributed (Gillespie, 2007). One of the most straightforward numerical Monte Carlo methods for generating simulated trajectories of $\mathbf{Z}(t)$ is the first reaction method described in Gillespie (1976). The idea behind the method is to generate $M$ prospective event times $\tau_{j}$ by sampling the exponential distribution with rate constant $a_{j}(\mathbf{z})$ and then choose the one that occurs first. The basic algorithm is as follows:

0 . Initialize the time $t=t_{0}$ and the initial state $\mathbf{z}=\mathbf{z}_{o}$.

1. Evaluate the propensity functions $a_{j}(\mathbf{z})$.

2. Draw $M$ random variables $r_{1}, \ldots, r_{M}$ on $(0,1)$.

3. Generate prospective reaction times $\tau_{j}=\frac{-\ln \left(r_{j}\right)}{a_{j}(\mathbf{z})}, j=1, \ldots, M$.

4. Take $\tau_{m}=\min \left\{\tau_{j}\right\}$.

5. Update the system state: $t \leftarrow t+\tau_{m}$ and $\mathbf{z} \leftarrow \mathbf{z}+\boldsymbol{v}_{m}$.

6. Return to Step 1, or if reached desired simulation time, end the simulation.

This algorithm was used to generate sample realizations of the stochastic process and compute persistence probabilities for the population as a function of simulation time by averaging over multiple realizations. Analysis and a discussion of the implications of these results for populations in advective media will be postponed until Section 4 . Note that the first reaction method becomes inefficient for large $K$ due to explosive exponential growth, and thus requires the implementation of more advanced algorithms (Gillespie, 2007). In this paper, we restrict our study to populations undergoing density dependent growth with sufficiently small $K$.

\subsection{Simulating the movement dynamics}

Movement takes place as a position-jump process with dispersal distance drawn from the redistribution kernel $k(x, y)$. As discussed in Section 2.2, the kernel was derived from the 
fast-scale movement dynamics described by an advection-diffusion process with constant settlement rate $\alpha$. Thus, to determine the position of a relocating individual, we freeze the main population dynamics timestepper $\tau$, while simulating the movement dynamics as a biased random walk across the lattice of patches. For the movement dynamics at the individual-based level to correspond to an advection-diffusion process at the populationlevel, we set the time step $\Delta s=\Delta x^{2} / 2$, the probability of moving one patch to the right $q_{r}=\frac{1}{2}+\frac{\tilde{v}}{4} \Delta x$, and the probability of moving one patch to the left $q_{l}=\frac{1}{2}-\frac{\tilde{v}}{4} \Delta x$. For $\Delta s$ and $\Delta x$ sufficiently small, this closely approximates an advection-diffusion process (Bailey, 1964). To determine the number of steps $m$ of length $\Delta s$ an individual random walker takes, we sample the exponential distribution of stopping times $p(s)=\tilde{\alpha} e^{-\tilde{\alpha} s}$. A time $S$ may be sampled from the exponential distribution by drawing a random number $u$ on $(0,1)$ and taking $S=-\ln (u) / \tilde{\alpha}$. The number of steps $m$ in the random walk is then taken to be the nearest integer $m$ which satisfies $m \Delta s=S$. If an individual in patch $i$ relocates to patch $r$ at the end of the random walk, the population count is decremented by 1 in patch $i$ and incremented by 1 in patch $r$ via the state change vector $\boldsymbol{v}_{i}$.

We now discuss our implementation of the boundary conditions. Recall that the dispersal process contributes to mortality because we make the assumption that individuals can leave the domain boundary. First, consider the case where movement is not altered at the domain boundary. In this case, if at the end of the random walk an individual has left the domain boundary, it is removed from the population for the remainder of the simulation. Now, instead, consider the case of a domain with a source at the upstream end and a sink at the downstream end. We implement these boundary conditions as follows. If a random walker reaches the upstream boundary, it remains stationary with probability $q_{l}$ and moves one patch to the right with probability $q_{r}$. If an individual reaches the downstream boundary, it moves one patch to the left with probability $q_{l}$ and is removed from the simulation with probability $q_{r}$. This microscopic description of the movement dynamics agrees with the macroscopic model of movement as an advection-diffusion process with no-flux and absorbing boundary conditions (Bailey, 1964; Lutscher et al., 2006).

\subsection{Packet dispersal}

In our current model of dispersal, there is no correlation in individual movement. Dispersal distances are independent and identically-distributed (iid) random variables. However, in some cases, it may be more reasonable to assume that there are correlations in dispersal. For example, the dispersal of larvae in the coastal ocean may be driven by stochastic eddy events which carry many individuals in "packets" from one location to another (Siegel et al., 2008). Another good example is wind dispersed seed pods.

Define $\tau_{c}$ as the timescale over which the interpatch movement of residents is correlated. To simulate correlations in dispersal, at the beginning of the time window $\tau_{c}$, $N$ iid dispersal distances are drawn from the redistribution kernel $k(x, y)$ so that there is a single dispersal distance associated with each patch. This sets up a fixed dispersal network of activity across patches. We refer to this as "packet" dispersal since over the time window $\tau_{c}$, individuals from a given patch are subject to the same dispersal conditions, and thus on a coarser time scale can be thought of as moving in packets upstream or downstream (or remain stationary) of their initial starting location. In the next section, we will quantify the effects of packet dispersal on population persistence as a function of the correlation time $\tau_{c}$. 
Table 1 Summary of dimensionless model parameters

\begin{tabular}{lll}
\hline Parameter & Symbol & Values explored \\
\hline Carrying capacity & $K$ & $500-5000$ \\
Intrinsic growth rate & $\tilde{r}$ & 1 \\
Dispersal rate & $\tilde{\mu}$ & 50 \\
Settlement rate & $\tilde{\alpha}$ & 1000 \\
Domain length & $\tilde{L}$ & 2 \\
Patch size & $\Delta x$ & $0.01-0.05$ \\
Diffusivity & $\tilde{D}$ & 1 \\
Flow speed & $\tilde{v}$ & $7.9-9.2$ \\
Correlation time & $\tau_{c}$ & $0-0.2$ \\
\hline
\end{tabular}

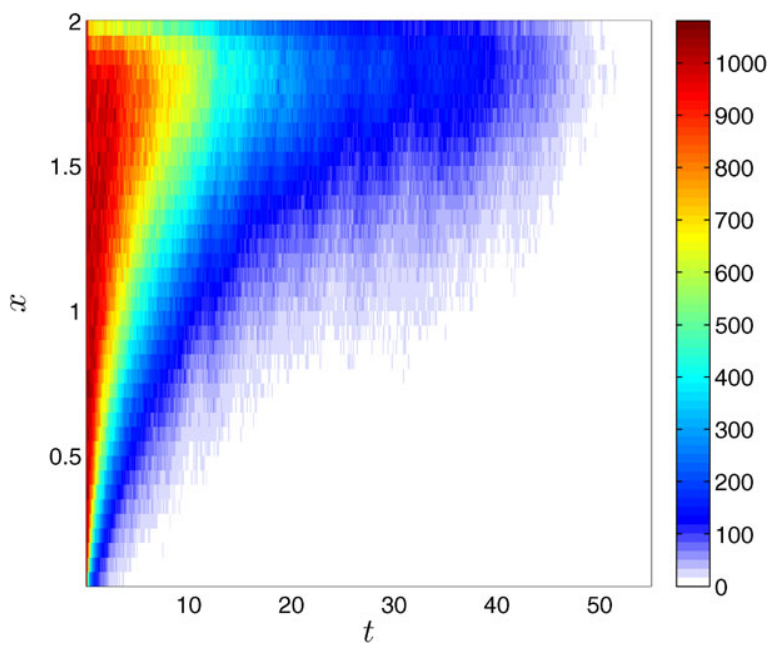

Fig. 2 Sample realization of the spatio-temporal dynamics of a population initially at carrying capacity on a domain of length $\tilde{L}=2$ with a no-flux boundary at the upstream end $(x=0)$ and an absorbing boundary at the downstream end $(x=2)$. The flow speed, $\tilde{v}=9.2$, is above the critical flow speed $\tilde{v}_{c}=8.6$. After an initial growth phase, the population goes extinct in approximately 50 time units.

\section{Results}

\subsection{The effects of demographic stochasticity}

To quantify the effects of stochasticity on persistence, multiple realizations of the model were performed using the stochastic simulation algorithm to simulate sequentially in continuous time, birth, death, and relocation events. See Table 1 for a summary of the parameters used in the simulations. Note that for the rest of our analysis, we will focus on the case of a domain with a source at the upstream end and a sink at the downstream end. However, the results are similar for Dirichlet boundary conditions. Figure 2 shows a sample realization of the process on a domain with a source at the top and a sink at the bottom at a velocity exceeding the critical value. After an initial transient in which the population grows in size, it eventually decays and goes extinct after approximately 50 time units. 


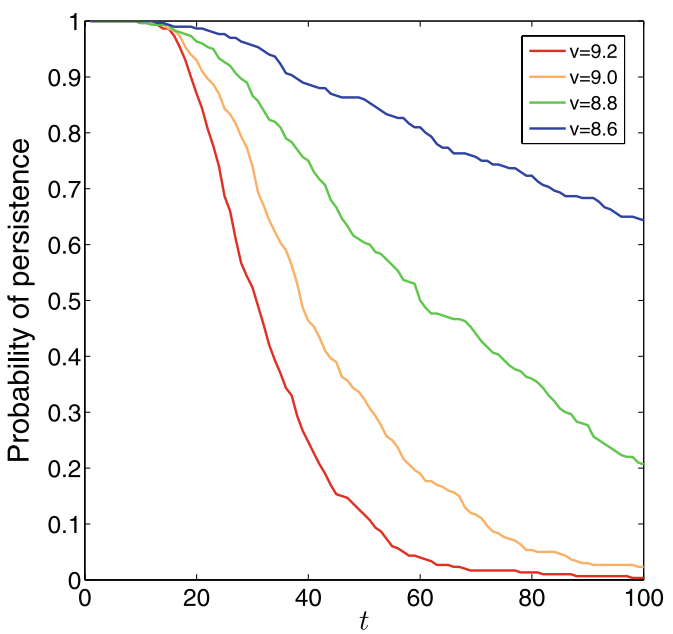

Fig. 3 Probability of persistence as a function of time for four different flow speeds: (blue) $\tilde{v}=8.6$, (green) $\tilde{v}=8.8$, (orange) $\tilde{v}=9.0$, (red) $\tilde{v}=9.2$. (Color figure online.)

Persistence probability time series were computed by averaging the stochastic simulation results across three hundred realizations. Figure 3 shows the time dependence of the probability of persistence of a population initially comprised of forty individuals for various flow speeds. After an initial transient, the persistence curves decay exponentially, with a more rapid decay as $\tilde{v}$ is increased. This is in accordance with what one would expect from a simple birth-death process where the intrinsic growth rate is nonpositive (Allen, 2003; Nisbet and Gurney, 2003). This makes sense because the solution to the linearized integrodifferential equation (10) can be written in an eigenfunction expansion as

$$
p(x, t)=\sum_{n=1}^{\infty} a_{n} e^{\lambda_{n} t} \phi_{n}(x)
$$

with eigenvalues $\lambda_{n}=1-\tilde{\mu}+\tilde{v}_{n}$ and eigenfunctions $\phi_{n}(x)$. Note that $\tilde{v}_{n}$ are the eigenvalues associated with the integral operator in (10). Averaging across the spatial variable, it follows that the rate of decay of solutions in time is the sum of exponentials, and thus controlled by the principle eigenvalue. Indeed, estimates of the decay rates of the persistence curves agree to within a factor of two of the principle eigenvalue associated with the eigenfunction expansion; see Table 2 . Since the eigenvalues vary smoothly with the flow speed $\tilde{v}$, it is no surprise that there is a continuous transition in persistence probability as $\tilde{v}$ is varied; see Fig. 4 .

Figure 4 shows the persistence probability after 100 time units as a function of $\tilde{v}$ for three different birth and death rates: (1) $\tilde{b}_{1}=2.0, \tilde{d}_{1}=1.0$, (2) $\tilde{b}_{1}=3.0, \tilde{d}_{1}=2.0$, and (3) $\tilde{b}_{1}=4.0, \tilde{d}_{1}=3.0$. All three cases correspond to the same deterministic model since $\tilde{r}=\tilde{b}_{1}-\tilde{d}_{1}=1.0$. The persistence probability curves decrease as $\tilde{b}_{1}$ and $\tilde{d}_{1}$ are increased simultaneously keeping $\tilde{b}_{1}-\tilde{d}_{1}$ constant. We expect such a decrease in persistence to a given time since increasing the turnover rates increases the variability in production. Note that the persistence probability curves become more jagged as $\tilde{b}_{1}$ is increased. This is 
Table 2 Comparison of decay rates in deterministic and stochastic model

\begin{tabular}{lll}
\hline Flow speed $(\tilde{v})$ & Estimate from persistence curves & Principle eigenvalue $(1-\tilde{\mu}+\tilde{v})$ \\
\hline 8.6 & -0.0055 & 0 \\
8.7 & -0.0157 & -0.0234 \\
8.8 & -0.0287 & -0.0431 \\
8.9 & -0.0356 & -0.0642 \\
9.0 & -0.0483 & -0.0847 \\
9.1 & -0.0573 & -0.1053 \\
9.2 & -0.0964 & -0.1271 \\
\hline
\end{tabular}

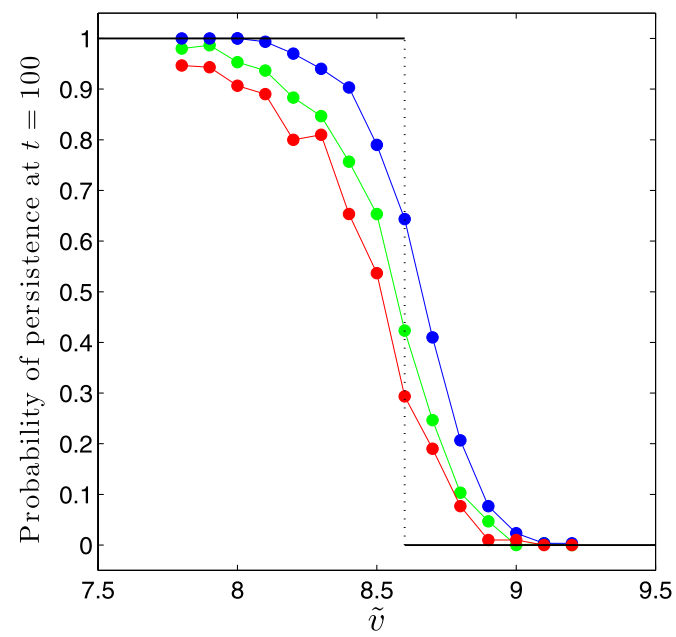

Fig. 4 Probability of persistence after 100 time units as a function of $\tilde{v}$ with $\tilde{L}=2$. The parameters are $\Delta x=0.05, K=1000, \tilde{\mu}=50, \tilde{\alpha}=1000$, and $\tilde{r}=1$ and the results were averaged over 300 replicates. The effects of demographic stochasticity were explored by increasing $b_{1}$ and $d_{1}$ while maintaining $\tilde{r}$. We consider three cases: (blue) $b_{1}=2.0, d_{1}=1.0$, (green) $b_{1}=3.0, d_{1}=2.0$, and (red) $b_{1}=4.0, d_{1}=3.0$. The results of the deterministic theory are shown for comparison (black). (Color figure online.)

due to the fact that there is more variability in the population dynamics. Finally, we note some results regarding nonlinear effects. We find that decreasing the carrying capacity lowers the persistence probabilities uniformly across the range of flow speeds considered. We also find that the persistence probabilities are affected by the spatial scale of density dependence. Specifically, decreasing $\Delta x$, while scaling $K$ accordingly, decreases the persistence probabilities uniformly across $\tilde{v}$.

\subsection{The effects of packet dispersal on persistence}

We explored the effects of packet dispersal on persistence by simulating the model for a wide range of correlation times $\tau_{c}$ and different flow speeds $\tilde{v}$. We consider correlation times that are up to a factor of ten times the mean time between dispersal events $t_{d}=$ $1 / \tilde{\mu}=0.02$. In all cases, we find that correlations in the dispersal activity of individuals can have a large positive impact on persistence. In Fig. 5, we compare the persistence 


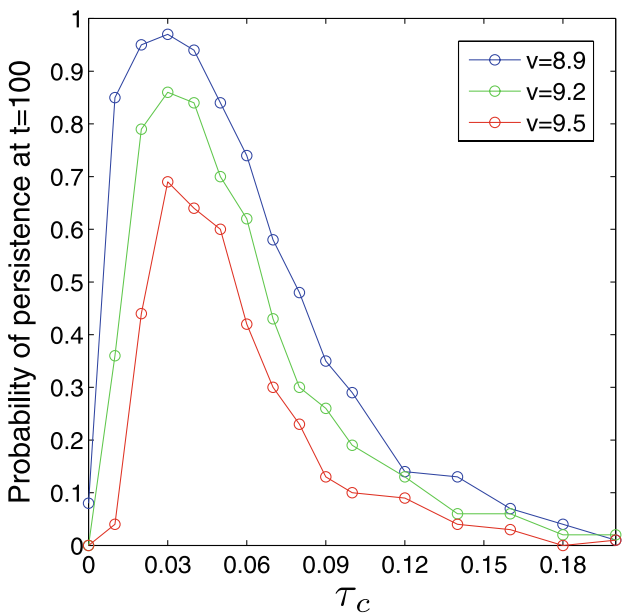

Fig. 5 Probability of persistence after 100 time units as a function of correlation time $\tau_{c}$. The parameters are $\tilde{L}=2, \Delta x=0.05, K=1000, \tilde{\mu}=50, \tilde{\alpha}=1000$, and $\tilde{r}=1$ and the results were averaged over 300 replicates. Here, we compare the impact of introducing a correlation in dispersal patterns on three different flow speeds: (blue) $\tilde{v}=8.9$, (green) $\tilde{v}=9.2$, and (red) $\tilde{v}=9.5$. (Color figure online.)

probability after 100 time units as a function of $\tau_{c}$ for three different flow speeds. Each data point was averaged over three hundred realizations of the stochastic model. In all cases, packet dispersal has the greatest impact on persistence when the correlation time is of similar magnitude to $t_{d}$. For example, when $\tilde{v}=8.9$, the probability of persistence goes from 0.08 when $\tau=0$ (no correlation in dispersal activity) to a maximum of 0.97 when $\tau_{c}=0.03$. For larger flow speeds, the trend is the same, but the magnitude of effect decreases with $\tilde{v}$.

We can understand the effects of packet dispersal on population persistence as follows. When $\tau_{c} \geq t_{d}$, individuals from a particular patch complete on average at least one jump to the same destination before the dispersal network changes. At the coarse-grained level, this corresponds to a single dispersal event carrying a packet of "meta-individuals" from one patch to another. When $\tau_{c} \approx t_{d}$, the dispersal network changes after on average one jump so that there is no correlation with the previous dispersal events from that patch. This corresponds to the case in which samples of the local wind or flow speed and direction are uncorrelated on the timescale of $\tau_{c}$ steps. Figure 6 shows a sample of the population dynamics when $\tau_{c}=0.03$, which is the peak in response of the model to the introduction of correlations in dispersal activity. At the coarse-grained level, meta-individuals undergo a biased random walk, just as individuals do in the original model. Dispersal events carry entire packets (as opposed to single individuals) downstream, upstream, or leave them stationary with respect to their initial location. Unlike single individuals, meta-individuals are able to maintain a firmer foothold upstream, since the more individuals there are, the less of an effect demographic stochasticity has on the population dynamics.

When $\tau_{c} \gg t_{d}$, the dispersal network remains fixed as packets move from patch to patch. Because of this, one may no longer think of the packets of meta-individuals as undergoing a biased random walk. Instead, meta-individuals are subject to the dynamics imposed by the fixed dispersal network. Indeed, if $\tau_{c}$ is sufficiently large so that packets 

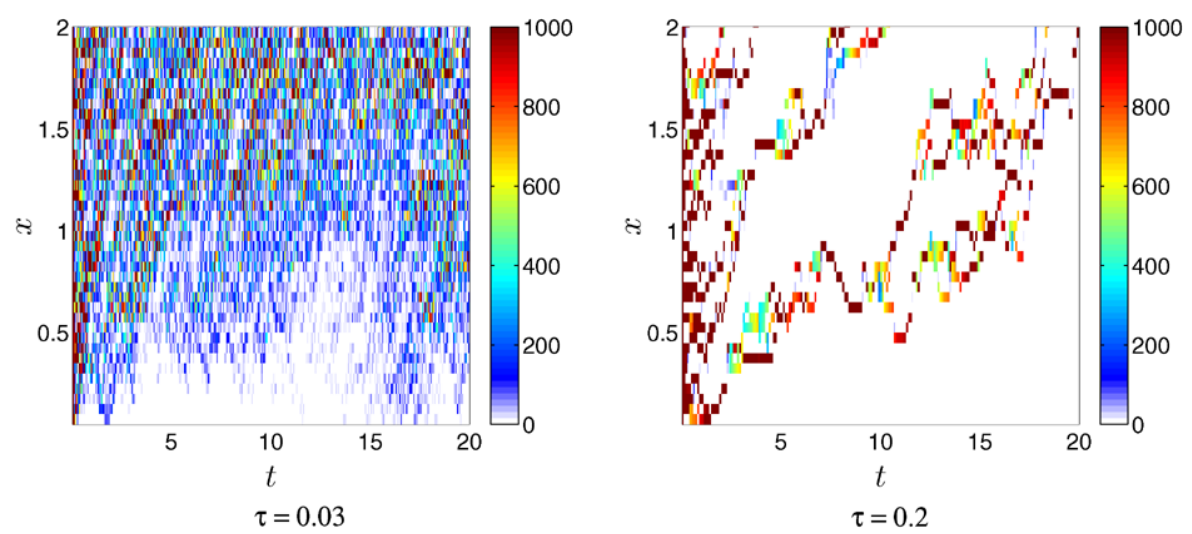

Fig. 6 Sample realizations of the stochastic process with correlation time (left) $\tau=0.03$ and (right) $\tau=0.2$. Parameters: $\tilde{L}=2, \Delta x=0.05, \tilde{b}_{1}=2, \tilde{d}_{1}=1, \tilde{\mu}=50, \tilde{\alpha}=1000, K=1000, \tilde{v}=9.2$, and $\tilde{v}_{c}=8.6$. No-flux boundary at the top of the domain and absorbing boundary at the bottom.

are carried along multiple time steps in the network, they will eventually settle to local sinks. When the meta-individuals have been carried a sufficient distance downstream, they are absorbed at the downstream end. This can be clearly seen in the second panel of Fig. 6.

\section{Discussion}

We have introduced a stochastic model for population dynamics in advective media to explore the effects of demographic stochasticity, both in reproduction and movement, on the population dynamics. When there are no correlations in dispersal activity, we observe a continuous transition in probability from persistence to extinction as the flow speed $\tilde{v}$ is varied. In the physics literature, this smooth transition from one state (persistence) to another state (extinction) is referred to as a second order or continuous phase transition and has been noted in a wide range of spreading processes which transition into an absorbing state (Hinrichsen, 2000).

Our results show that the deterministic theory is quite robust to noise effects: demographic stochasticity simply smooths the persistence and extinction boundary and does not result in any catastrophic phenomena such as discontinuities in the transition. However, because of this smooth transition in persistence probability, there is a range of flow speeds $\tilde{v}<\tilde{v}_{c}$ for which the deterministic theory predicts persistence but the stochastic model indicates that the population may go extinct in a finite number of time units. Thus, demographic stochasticity can lead to the extinction of a population, even under conditions for which the deterministic theory implies that a population will survive indefinitely. This is something that should be kept in mind if critical domain size estimates are invoked in environmental management.

Complementary studies on the effects of demographic stochasticity on invasion speeds have been done in Kawasaki et al. (2006), Kot et al. (2004), Lewis (2000), Mollison 
(1977), Snyder (2003). For linear systems on an infinite domain, it was shown that stochasticity does not generally slow invasions. For nonlinear systems, variability in production and movement typically slows invasions, however, see Kawasaki et al. (2006) for an example where it speeds them up. It was noted in Pachepsky et al. (2005) that the asymptotic velocity above which no population can survive on any length of domain is equivalent to the critical speed in which an invasion front switches from advancing upstream to retreated downstream. Therefore, the predictions regarding invasion speeds may be interpreted as suggesting that for nonlinear models; stochasticity can result in a shift in the persistence and extinction boundary. In our study, for a domain of length $\tilde{L}=2$, a shift in the critical flow speed can be observed. Specifically, suppose we take the critical velocity in the stochastic model as the flow speed for which the population has a $50 \%$ probability of persistence. Then the critical velocity shifts to smaller values when the variability in production is increased or the carrying capacity is lowered. See Fig. 4 for an example of the former.

We have quantified the effects of introducing correlations in the dispersal activity of individuals on population persistence. Our findings indicate that when there is approximately no correlation in the sampled flow field from a given patch on the timescale that a packet moves from one patch to another, the movement of individuals in packets greatly enhances population persistence. This is due to the fact that packets of individuals are less susceptible to extinction than single individuals under the influence of demographic stochasticity. Our results provide insight into the mechanisms contributing to population persistence under conditions where dispersers are transported in packets such as larvae in the coastal ocean or wind-dispersed seed pods.

In Siegel et al. (2008), it was shown that packet dispersal may be the primary means of transport for fish and invertebrate populations in the coastal ocean with an obligate larval stage. The driving mechanism behind packet transport is coastal eddies which intermittently sweep larvae from one location to another in settlement pulses. The source and destination of these settlement pulses is highly variable within each season and across multiple spawning seasons leading to heterogeneity in connectivity among subpopulations. This same mechanism of transport is built into our continuous-time model when we assume there is no correlation in the destination of individuals from a given patch on the timescale in which a packet moves from one patch to another.

It was demonstrated in Berkley et al. (2010) and in work by Mitarai et al. highlighted in a review article by Cowen and Sponaugle (2009) that the dispersal of individuals in packets can bring about species coexistence under conditions which would otherwise preclude it. In the study highlighted in Cowen and Sponaugle (2009), an idealized coastal circulation model was coupled with a Lagrangian particle tracking model to simulate the transport of larvae in packets in the coastal ocean. The population dynamics were formulated in discrete time using a Beverton-Holt competition model. In the simulations, one species was initially introduced upstream and the other downstream. When dispersal was simulated as a simple advection-diffusion process, only the upstream species ultimately persisted. In contrast, the dispersal of individuals in packets promoted coexistence. The ability of subpopulations to settle upstream was the driving mechanism behind this result, allowing groups of downstream individuals to get a foothold in less densely occupied spaces upstream. The same mechanism is at work in our single-species model, allowing populations to persist over much longer timescales. 


\section{Acknowledgements}

We thank Kurt Anderson, Bruce Kendall, Frithjof Lutscher, Satoshi Mitarai, and David Siegel for valuable discussions. The research was supported by grants from the US National Science Foundation (DEB 0717259) to R.M.N. and CALFED (U-05-SC-058) to Dr. Tom Dunne.

Open Access This article is distributed under the terms of the Creative Commons Attribution Noncommercial License which permits any noncommercial use, distribution, and reproduction in any medium, provided the original author(s) and source are credited.

\section{References}

Alexander, S.E., Roughgarden, J., 1996. Larval transport and population dynamics of intertidal barnacles: a coupled benthic/oceanic model. Ecol. Monogr. 66, 259-275.

Allen, L.J.S., 2003. An Introduction to Stochastic Models with Applications to Biology. Prentice Hall, New York.

Anderson, K.E., Paul, A.J., McCauley, E., Jackson, L.J., Post, J.R., Nisbet, R.M., 2006. Instream flow needs in streams and rivers: the importance of understanding ecological dynamics. Front. Ecol. Environ. 4(6), 309-318.

Bailey, N.T.J., 1964. The elements of Stochastic Processes. Wiley, New York.

Ballyk, M., Smith, H., 1999. A model of microbial growth in a plug flow reactor with wall attachment. Math. Biosci. 158(2), 95-126.

Berkley, H.A., Kendall, B.E., Mitarai, S., Siegel, D.A., 2010. Turbulent dispersal promotes species coexistence. Ecol. Lett., submitted.

Byers, J.E., Pringle, J.M., 2006. Going against the flow:retention, range limits and invasions in advective environments. Mar. Ecol. Prog. Ser. 313, 27-41.

Cowen, R.K., Sponaugle, S., 2009. Larval dispersal and marine population connectivity. Ann. Rev. Mar. Sci. 1(1), 443-466.

Elliott, J.M., 2002. Time spent in the drift by downstream-dispersing invertebrates in a lake district stream. Freshwater Biol. 47(1), 97-106.

Gaines, S.D., Bertness, M.D., 1992. Dispersal of juveniles and variable recruitment in sessile marine species. Nature 360, 579-580.

Gillespie, D.T., 1976. A general method for numerically simulating the stochastic time evolution of coupled chemical reactions. J. Comput. Phys. 22(4), 403-434.

Gillespie, D.T., 2007. Stochastic simulation of chemical kinetics. Ann. Rev. Phys. Chem. 58, 35-55.

Gurney, W.S.C., Nisbet, R.M., 1998. Ecological Dynamics. Oxford University Press, New York.

Hershey, A.E., Pastor, J., Peterson, B., Kling, G.W., 1993. Stable isotopes resolve the drift paradox for baetis mayflies in an arctic river. Ecology 74, 2315-2325.

Hinrichsen, H., 2000. Nonequilibrium critical phenomena and phase transitions into absorbing states. Adv. Phys. 49(7), 815-958.

Humphries, S., Ruxton, G.D., 2002. Is there really a drift paradox? J. Animal Ecol. 71, 151-154.

Kawasaki, K., Takasu, F., Caswell, H., Shigesada, N., 2006. How does stochasticity in colonization accelerate the speed of invasion in a cellular automaton model? Ecol. Res. 21, 334-345.

Kot, M., Medlock, J., Reluga, T., Walton, D.B., 2004. Stochasticity, invasions, and branching random walks. Theor. Popul. Biol. 66, 175-184.

Lewis, M.A., 2000. Spread rate for a nonlinear stochastic invasion. J. Math. Biol. 41, 430-454.

Lutscher, F., Pachepsky, E., Lewis, M.A., 2005. The effect of dispersal patterns on stream populations. SIAM Rev. 47(4), 749-772.

Lutscher, F., Lewis, M.A., McCauley, E., 2006. Effects of heterogeneity on spread and persistence in rivers. Bull. Math. Biol. 68(8), 2129-2160.

Mollison, D., 1977. Spatial contact models for ecological and epidemic spread. J. R. Stat. Soc. B 39, 238-326.

Müller, K., 1974. Stream drift as a chronobiological phenomenon in running water ecosystems. Ann. Rev. Ecol. Syst. 5, 309-323. 
Müller, K., 1982. The colonisation cycle of freshwater insects. Oecologia 52, 202-207.

Nisbet, R.M., Gurney, W.C.S., 2003. Modelling Fluctuating Populations. Blackburn Press.

Okubo, A., 1980. Diffusion and Ecological Problems: Mathematical Models. Springer, Berlin.

Pachepsky, E., Lutscher, F., Nisbet, R.M., Lewis, M.A., 2005. Persistence, spread and the drift paradox. Theor. Popul. Biol. 67(1), 61-73.

Siegel, D.A., Mitarai, S., Costello, C.J., Gaines, S.D., Kendall, B.E., Warner, R.R., Winters, K.B., 2008. The stochastic nature of larval connectivity among nearshore marine populations. PNAS 105(26), 8974-8979.

Skellam, J.G., 1951. Random dispersal in theoretical populations. Biometrika 38, 196-218.

Snyder, R.E., 2003. How demographic stochasticity can slow biological invasions. Ecology 84(5), 13331339.

Speirs, D.C., Gurney, W.S.C., 2001. Population persistence in rivers and estuaries. Ecology 82(5), 12191237. 